\title{
Desquamative interstitial pneumonitis and systemic lupus erythematosus
}

\author{
Faranak Esmaeilbeigi HBSc ${ }^{1}$, Stephen Juvet MD FRCPC ${ }^{2}$, David Hwang MD PhD FRCPC ${ }^{2}$, Shikha Mittoo MD MHS FRCPC ${ }^{2}$
}

\begin{abstract}
F Esmaeilbeigi, S Juvet, D Hwang, S Mittoo. Desquamative interstitial pneumonitis and systemic lupus erythematosus. Can Respir J 2012;19(1):50-52.

Desquamative interstitial pneumonia (DIP) is a rare form of interstitial lung disease (ILD) commonly found among healthy smokers. ILD is a rare manifestation of systemic lupus erythematosus (SLE), and typically associated with a histopathological pattern of nonspecific interstitial pneumonia (NSIP). The present article describes an unusual case of DIP in a nonsmoking patient with SLE presenting as NSIP. DIP can occur in the context of SLE in patients with a negative smoking history, and clinicians should consider lung biopsy to correctly classify ILD with unusual presentation on computed tomography scan.
\end{abstract}

Key Words: Connective tissue disease; Interstitial lung disease; Lupus

\section{Learning objectives}

-To recognize the potential importance of a surgical lung biopsy in selected patients with connective tissue disease.

-To understand the histopathological and radiological appearance of desquamative interstitial pneumonia.

CanMEDS competency: Medical expert

Pre-test

- When should surgical lung biopsy be considered in a patient with high-resolution computed tomography findings consistent with interstitial lung disease?

- Why is it important to differentiate between nonspecific interstitial pneumonia and desquamative interstitial pneumonia?

\section{CASE PRESENTATION}

In September 2007, a 23-year-old man presented with a three-week history of symptoms suggestive of an upper respiratory tract infection (sneezing, mild dyspnea on exertion), lymphadenopathy and a sore throat without cough. In addition, he experienced low-grade fever for one day. He had no travel history or obvious sick contacts. He had no significant occupational exposure to respiratory irritants through his job in a security firm. He was a lifelong nonsmoker, and denied significant secondhand smoke exposure and use of illicit drugs. He was treated with clindamycin $300 \mathrm{mg}$ once daily for one week, and his symptoms resolved. Over the ensuing weeks, he developed what he described as a mild feeling of dyspnea that did not limit his daily activities and improved with deep breathing.

The patient's medical history was notable only for a diagnosis of systemic lupus erythematosus (SLE) made in 2001 based on the following features: polyarthritis, pleurisy, leucopenia, the presence of antinuclear antibody (ANA) at a titre of 1:640 and the presence of antidouble-stranded DNA (anti-dsDNA) (1). Previous autoantibody screening was negative for anticardiolipin/antiphospholipid antibodies, anti-Ro, anti-Sm, anti-La, anti-RNP, anti-Jo1 and anti-Scl 70 antibodies. He had no family history of lung disease or autoimmune disease.

Since his SLE diagnosis, he was maintained on hydroxychloroquine at a dose of $400 \mathrm{mg}$ per day, and required no other medications on a regular basis. His disease course was complicated only by intermittent episodes of pleuritic chest pain that prompted evaluation with a

\section{Une pneumonite interstitielle desquamative et un} lupus érythémateux disséminé

La pneumonite interstitielle desquamative (PID) est une forme rare de maladie pulmonaire interstitielle (MPI), souvent observée chez les fumeurs en bonne santé. La MPI est une rare manifestation de lupus érythémateux disséminé (LÉD), et elle s'associe généralement à un profil histopathologique de pneumonie interstitielle non spécifique(PINS). Le présent article décrit un cas inhabituel de PID chez un patient qui n'avait jamais fumé et qui avait un LÉD se présentant sous forme de PINS. La PID peut se manifester en cas de LÉD chez les patients n'ayant jamais fumé, et les cliniciens devraient envisager une biopsie pulmonaire pour bien classer la MPI ayant une présentation inhabituelle à la tomodensitométrie.

high-resolution computed tomography (HRCT) scan of the chest in 2001, which revealed multiple small nodules ( $3 \mathrm{~mm}$ to $6 \mathrm{~mm}$ in size); these nodules remained stable on follow-up HRCT in January 2003 and October 2004. At the time of this presentation in September 2007, he did not have any other symptoms suggestive of his previous SLE flares, in particular, no joint pain. On physical examination during follow-up in October 2007, the patient appeared well and exhibited normal vital signs. He had fine, late bibasilar inspiratory crackles without signs of volume overload. His musculoskeletal, cardiac, abdominal and dermatological examination was unremarkable; there was no clubbing, signs of lymphadenopathy or peripheral edema.

Pulmonary investigations included pulmonary function tests (PFTs), chest computed tomography (CT), bronchoalveolar lavage (BAL) and surgical lung biopsy. PFTs demonstrated a total lung capacity of $5.2 \mathrm{~L}$ ( $80 \%$ predicted) and a reduced forced vital capacity (FVC) of $3.6 \mathrm{~L}$ ( $75 \%$ of predicted [normal $\geq 80 \%$ ]); there was no evidence of obstruction, with a forced expiratory volume in $1 \mathrm{~s}\left(\mathrm{FEV}_{1}\right) /$ FVC ratio of $85 \%$ (normal $\geq 70 \%$ ). His diffusing capacity for carbon monoxide (DLCO) was normal at $80 \%$ predicted.

A CT scan revealed bilateral cystic lucencies, and ground glass opacities (GGOs) throughout the lungs suggesting a diagnosis of nonspecific interstitial pneumonia (NSIP) (Figure 1). GGOs were most pronounced over the base of the right middle lobe. Pulmonary nodules that had been previously stable now appeared to be enlarged, and there was progressive peripheral consolidation in the lower lobes. A BAL was performed to exclude an infectious source and revealed Haemophilus influenzae. He was treated with clindamycin without improvement in his respiratory status. Based on the clinical presentation, PFTs and CT results, the patient's diagnosis was compatible with interstitial lung disease (ILD) and the radiographic findings suggested NSIP as the histopathological subtype.

In November 2007, because of the unusual radiological appearance of cystic lucencies, a video-assisted thoracoscopic lung biopsy was performed to confirm the suspicion of NSIP. Surprisingly, the biopsy revealed alveolar filling by macrophages and was consistent with desquamative interstitial pneumonia (DIP) (Figure 2). He was initiated on $50 \mathrm{mg}$ of daily prednisone along with trimethoprim/sulfamethoxazole for Pneumocystis jiroveci prophylaxis. His prednisone was tapered by $10 \mathrm{mg}$ every six weeks, until he was at $30 \mathrm{mg}$, and was then tapered to $25 \mathrm{mg}, 20 \mathrm{mg}, 10 \mathrm{mg}, 5 \mathrm{mg}$ and, finally, $2.5 \mathrm{mg}$ at six-week intervals. Prednisone as monotherapy continued until December 2008. Throughout this time, his PFTs and bloodwork were monitored

${ }^{1}$ Department of Medicine, University of Western Ontario, London; ${ }^{2}$ Department of Medicine, University of Toronto, Toronto, Ontario

Correspondence: Mr Faranak Esmaeilbeigi, Department of Medicine, University of Western Ontario, London, Ontario N6A 5C1.

Telephone 647-785-8448, e-mail faranak.esmaeilbeigi@mail.utoronto.ca 

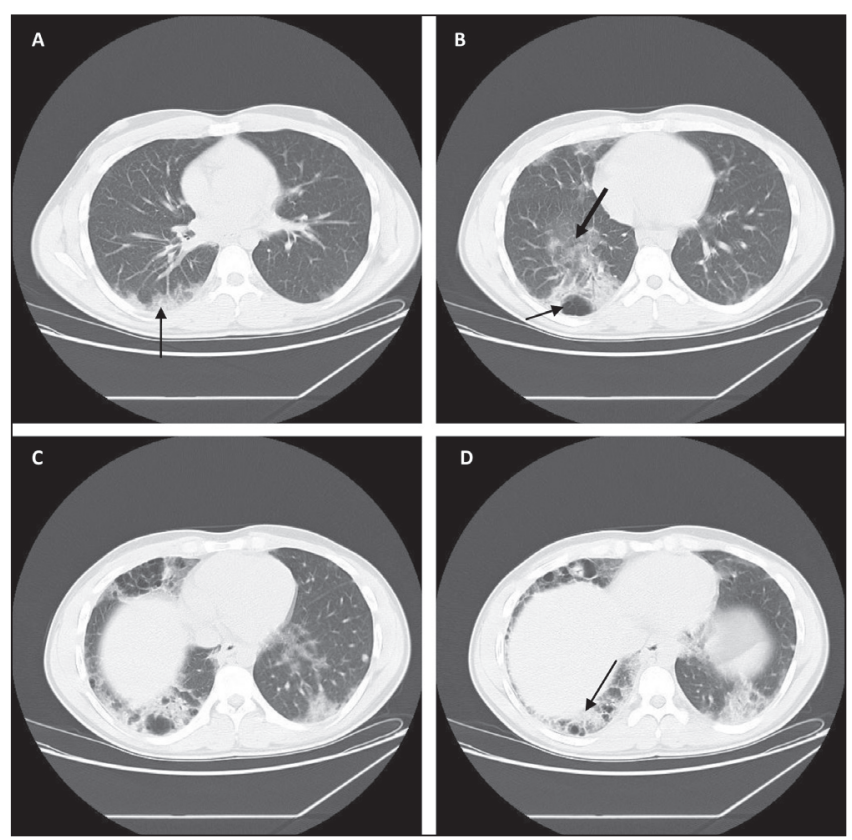

Figure 1) Computed tomography scan at presentation. A Faint opacities in right middle lobe with ground-glass opacity and more dense consolidation in the superior segment of the right lower lobe (arrow). B to D Peripheral consolidation, ground-glass opacities (thick arrow), cystic lucencies (thin arrow) in right and left lower lobe

approximately every three to six months to examine for lupus flares or respiratory deterioration. He underwent repeat HRCT scans performed in April 2008 that suggested improvement in his condition, and an additional HRCT scan in October 2008 that revealed new parenchymal abnormalities suggestive of recrudescence of his DIP. However, the patient remained asymptomatic, and physical examination findings were unremarkable.

In February 2009, shortly after his course of prednisone was completed, the patient developed worsening dyspnea on exertion. An HRCT scan of the chest revealed an increased number of GGOs in the posterior basilar segments of both lobes; his PFTs demonstrated normal lung function, with an FVC of $4.9 \mathrm{~L}$ (92\% predicted), total lung capacity of $5.9 \mathrm{~L}$ ( $88 \%$ predicted) and DLCO of $25.8 \mathrm{~L}$ (79\% predicted). BAL fluid was negative for infection or malignancy. In addition to his daily regimen of plaquenil, the patient was restarted on $50 \mathrm{mg}$ prednisone with the addition of azathioprine, titrated to a dose of $150 \mathrm{mg} / \mathrm{day}$, and trimethoprim/sulfamethoxazole. Pulmonary function tests remained stable, and a follow-up HRCT in July 2009 noted improvement in GGOs without change in cyst size or appearance. Over the ensuing 11 months, prednisone was tapered to $3 \mathrm{mg} /$ day with resolution of his symptoms.

\section{DISCUSSION}

Pulmonary involvement is common in SLE, occurring in up to $50 \%$ of patients. Despite this, the presence of ILD is rare in SLE (2). ILDs are a well-described complication of connective tissue diseases and encompass a heterogeneous group of disorders with various etiologies (3). Histopathological patterns include NSIP, usual interstitial pneumonia (UIP) and DIP (3-6). NSIP is the most common form of ILD seen in SLE. In contrast, DIP is believed to be exclusively linked to smoking $(4,7,8)$. DIP has been reported in nonsmoking patients with rheumatoid arthritis, polymyositis and systemic scleroderma (3-5). We reported an unusual case of DIP occurring as a complication of SLE that presented as NSIP.

Only one previous case of SLE-DIP has been described in a nonsmoking patient (9). In our patient, nonspecific pulmonary nodules were found early in the patient's course, before radiographic
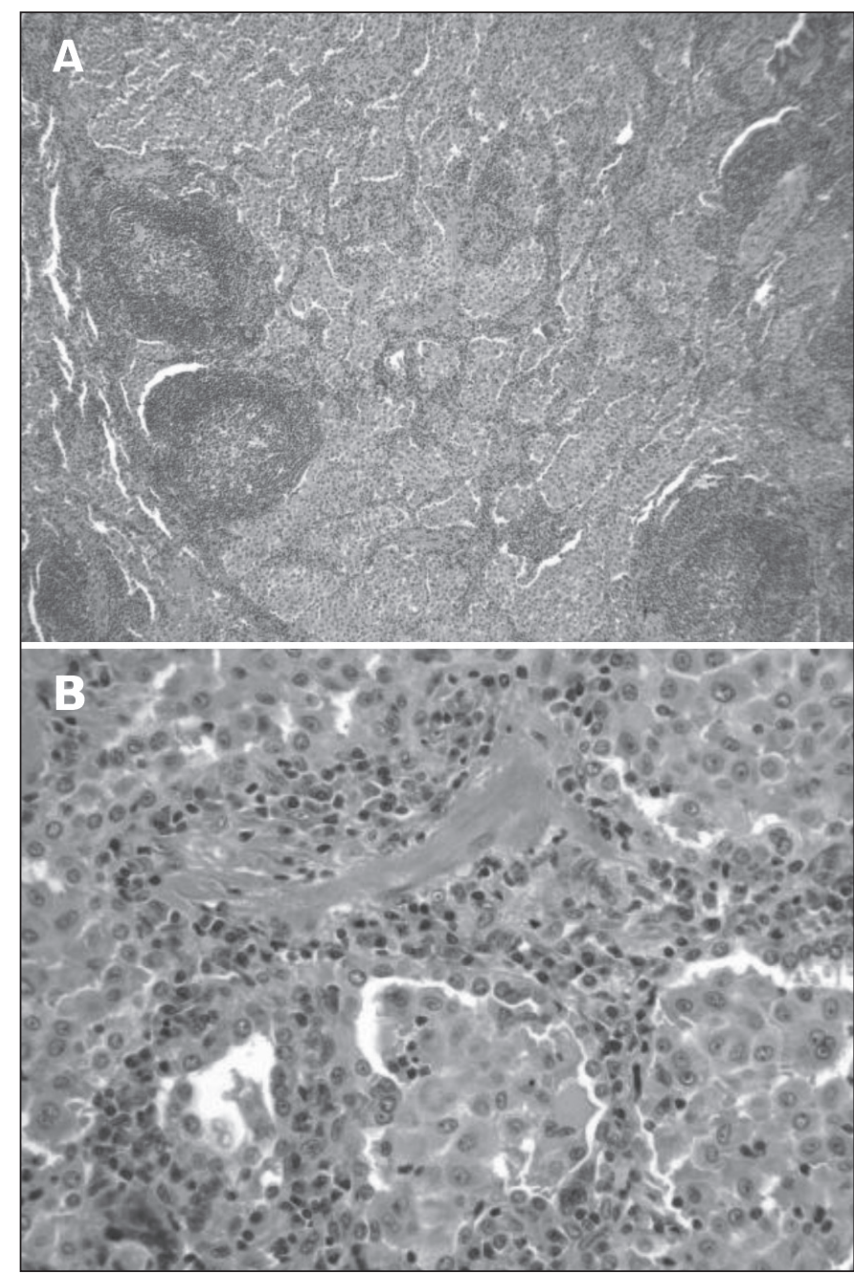

Figure 2) Histology of lung tissue obtained by video-assisted thoracoscopic lung biopsy. A Portion of lung tissue showing intra-alveolar filling with sheets of finely pigmented macrophages, associated with mild interstitial chronic inflammatory infiltrates and scattered lymphoid follicles, consistent with desquamative interstitial pneumonitis (hematoxylin and eosin stain, original magnification $\times 50$ ). B Higher magnification view of intra-alveolar macrophages with adjacent interstitial lymphoplasmacytic infiltrates (hematoxylin and eosin stain, original magnification $\times 200$ )

findings compatible with ILD. There have been other reports of nonspecific nodules on chest imaging in SLE; however, the clinical significance of these findings is unclear (10). Whether these nodules represent an early form of ILD in SLE is unknown.

The current approach to the diagnosis of ILD is based on clinical, radiological and pathological data. Surgical lung biopsy is the most sensitive method for collecting accurate pathological data; however, this procedure is performed in less than $15 \%$ of patients $(6,11)$. While surgical lung biopsy should be performed on a case-by-case basis, and is not necessarily required for every patient, consideration of a surgical lung biopsy should be considered in unusual cases, especially in the context of underlying connective tissue disease. One study examining the accuracy of ILD diagnosis based on HRCT scans alone (11) reported that experts could only accurately diagnose a UIP pattern $50 \%$ of the time. Moreover, histopathological patterns may have a similar appearance on chest HRCT scans; UIP, which typically presents with honeycombing, can present with GGOs and cystic nodules that suggest NSIP (11). Furthermore, DIP may present with GGOs and appear similar to NSIP on HRCT and, therefore, the findings on HRCT may not be specific (12). Consequently, it is important to consider a biopsy, particularly among patients who present without honeycombing on HRCT (13). 
An accurate diagnosis of the histopathological subtype of ILD is important to guide prognosis and management (11). While DIP and NSIP may both be responsive to corticosteroids, the prognosis in patients with DIP is significantly better than NSIP (12). The 10-year survival rate of DIP is reported to be approximately $70 \%$, whereas the survival rate in NSIP can be as low as 35\% depending on the subclass of NSIP $(14,15)$. Furthermore, a case report in 2008 (16) suggested that DIP may be responsive to therapy with macrolides and, therefore, there may be additional treatment options for patients with DIP unresponsive to corticosteroid therapy. It is also possible that DIP and NSIP are spectrums of the same disease, with DIP progressing to NSIP as fibrosis increases; however, this relationship has yet to be revealed (12).

\section{CONCLUSION}

The present report described an unusual case of DIP in a never-smoking patient with SLE. Clinicians should consider a lung biopsy in patients with atypical radiographic presentation in patients with ILD.

\section{REFERENCES}

1. Tan EM, Cohen AS, Fries JF, et al. The 1982 revised criteria for the classification of systemic lupus erythematosus. Arthritis Rheum 1982;25:1271-7.

2. Torre $\mathrm{O}$, Harari S. Pleural and pulmonary involvement in systemic lupus erythematosus. Presse Med 2011;40:e19-29.

3. Lamblin C, Bergoin C, Saelens T, et al. Interstitial lung diseases in collagen vascular diseases. Eur Respir J Suppl 2001;32:69s-80s.

4. De Lauretis A, Veeraraghavan S, Renzoni E. Review series: Aspects of interstitial lung disease: Connective tissue disease-associated interstitial lung disease: How does it differ from IPF? How should the clinical approach differ? Chron Respir Dis 2011;8:53-82.

5. Swartz JS, Chatterjee S, Parambil JG. Desquamative interstitial pneumonia as the initial manifestation of systemic sclerosis. J Clin Rheum 2010;16:284-6.

6. British Thoracic Society. The diagnosis, assessment and treatment of diffuse parenchymal lung disease in adults. Thorax 1999;54:S1-S28.

7. Liebow AA, Steer A, Billingsley JG. Desquamative interstitial pneumonia. Am J Med 1965;39:369.

8. Corrin B, Price AB. Electron microscope studies in desquamative interstitial pneumonia associated with asbestos. Thorax 1972;27:324-31.

\section{Post-test}

- When should surgical lung biopsy be considered in a patient with HRCT findings consistent with ILD?

- Surgical lung biopsy should be considered in all patients with HRCT findings that are unusual. For example, in the current case, the patient presented with cysts, which is unusual for NSIP and UIP.

- Why is it important to differentiate between NSIP and DIP?

- Although both NSIP and DIP may be responsive to corticosteroids, the prognosis for patients with DIP is significantly better than the prognosis for NSIP (12). Furthermore, recent evidence suggests that DIP may be responsive to macrolide therapy (14).

9. Remenyik E, Meszaros C, Vezendi S, et al. Simultaneous occurrence of lupus erythematosus and desquamative interstitial alveolitis. Orv Hetil 1990;131:2043-5.

10. Mittoo SM, Fischer A, Strand V, Meehan R, Swigris JJ. Systemic lupus erythematosus-related interstitial lung disease. Current Rheum Rev 2010;6:99-107.

11. Lynch DA, Travis WD, Muller NL, et al. Review: Idiopathic interstitial pneumonias: CT features. Radiology 2005;236:10-21.

12. Tazelaar HD, Wright JL, Churg A. Review: Desquamative interstitial pneumonia. Histopath 2011;58:509-16.

13. Flaherty KR, Toews GB, Travis WD, et al. Clinical significance of histological classification of idiopathic interstitial pneumonia. Eur Respir J 2002;19:275-83.

14. Carbone R, Bottino G, Paredi P, Shah P, Meyer KC. Predictors of survival in idiopathic interstitial pneumonia. Eur Rev Med Pharmacol Sci 2010;14:695-704.

15. American Thoracic Society/European Respiratory Society International Multidisciplinary Consensus Classification of the idiopathic interstitial pneumonias. Am J Respir Crit Care Med 2002;165:277-304.

16. Knyazhitskiy A, Masson RG, Corkey R, Joiner J. Beneficial response to macrolide antibiotic in a patient with desquamative interstitial pneumonia refractory to corticosteroid therapy. Chest 2008;134:185-7.

The Canadian Respiratory Journal is now accepting submissions for a new Clinico-Pathologic Conferences series. These will be based on case presentations that illustrate important learning issues involving diagnosis and/or management decisions, and should be supported by images from appropriately applied diagnostic and/or prognostic testing which could include: 1) Lung function tests; 2) Exercise testing; 3) X-rays or computed tomography scans; 4) Ultrasound (including endobronchial ultrasound); 5) Positron emission tomography scans; or 6) Bronchoscopy/thoracoscopy.

All case reports appearing in the Canadian Respiratory Journal will conform to this format and manuscripts should be structured as described in the Instructions to Authors. A maximum of four images can be submitted and the number of references should not usually exceed 10 . The submission will be peer reviewed and may be edited by our editorial team. 


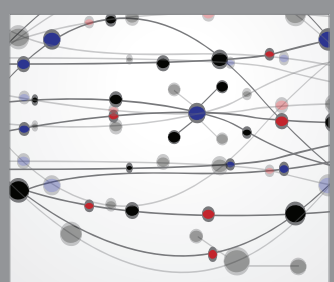

The Scientific World Journal
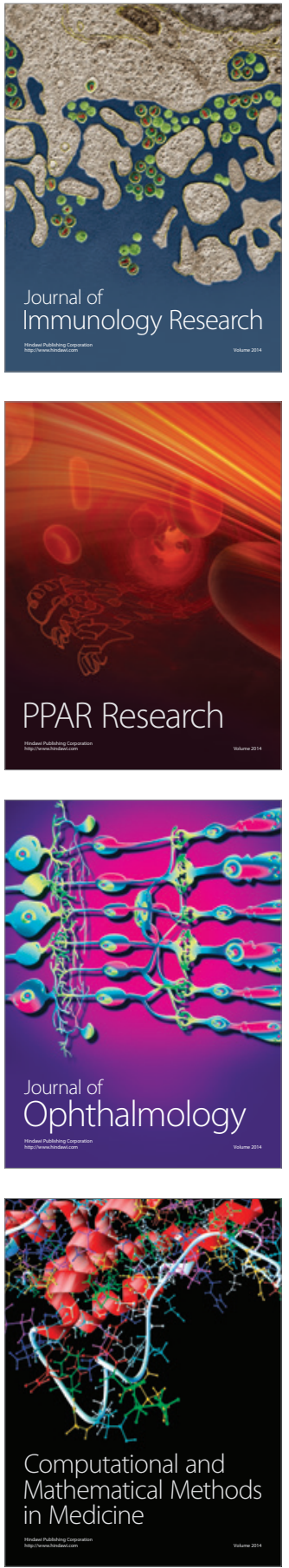

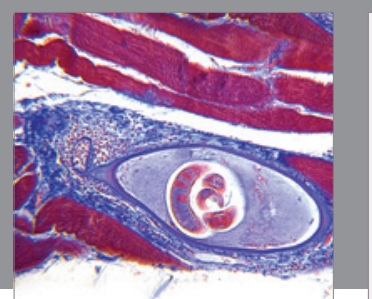

Gastroenterology Research and Practice

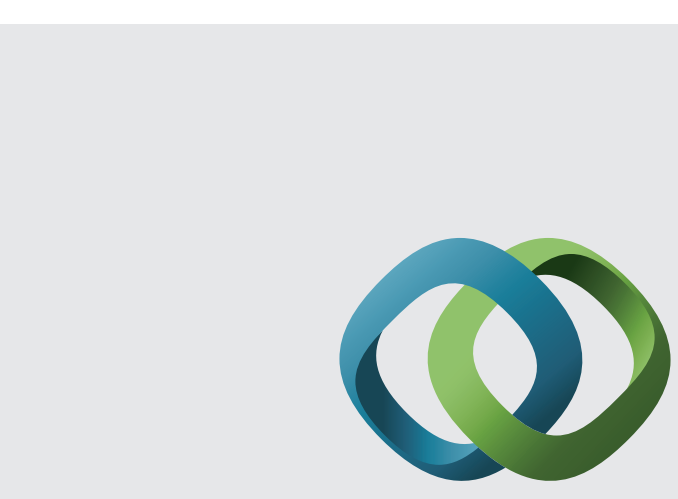

\section{Hindawi}

Submit your manuscripts at

http://www.hindawi.com
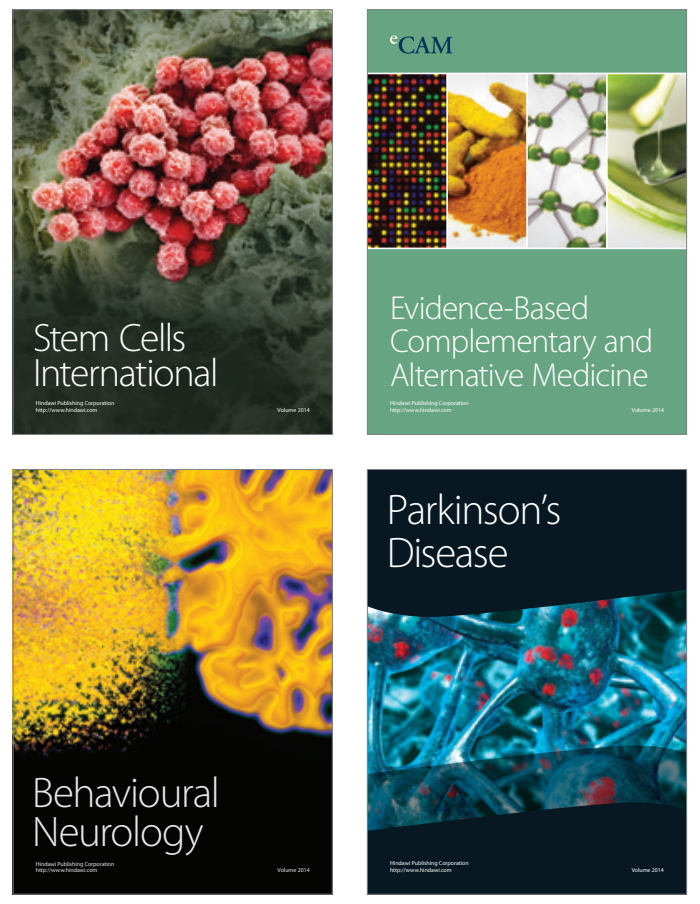
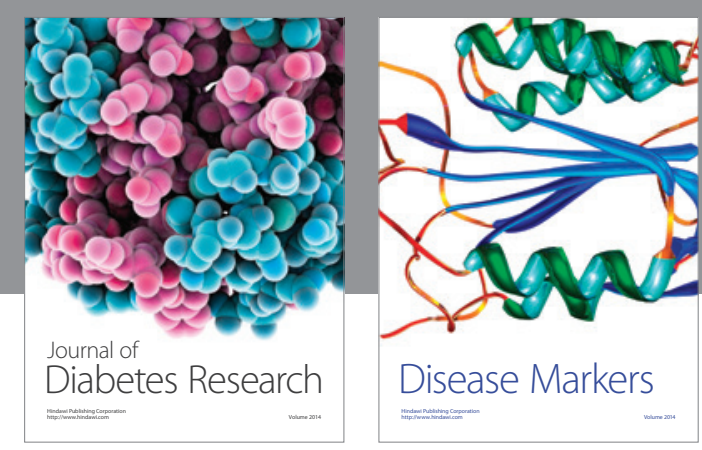

Disease Markers
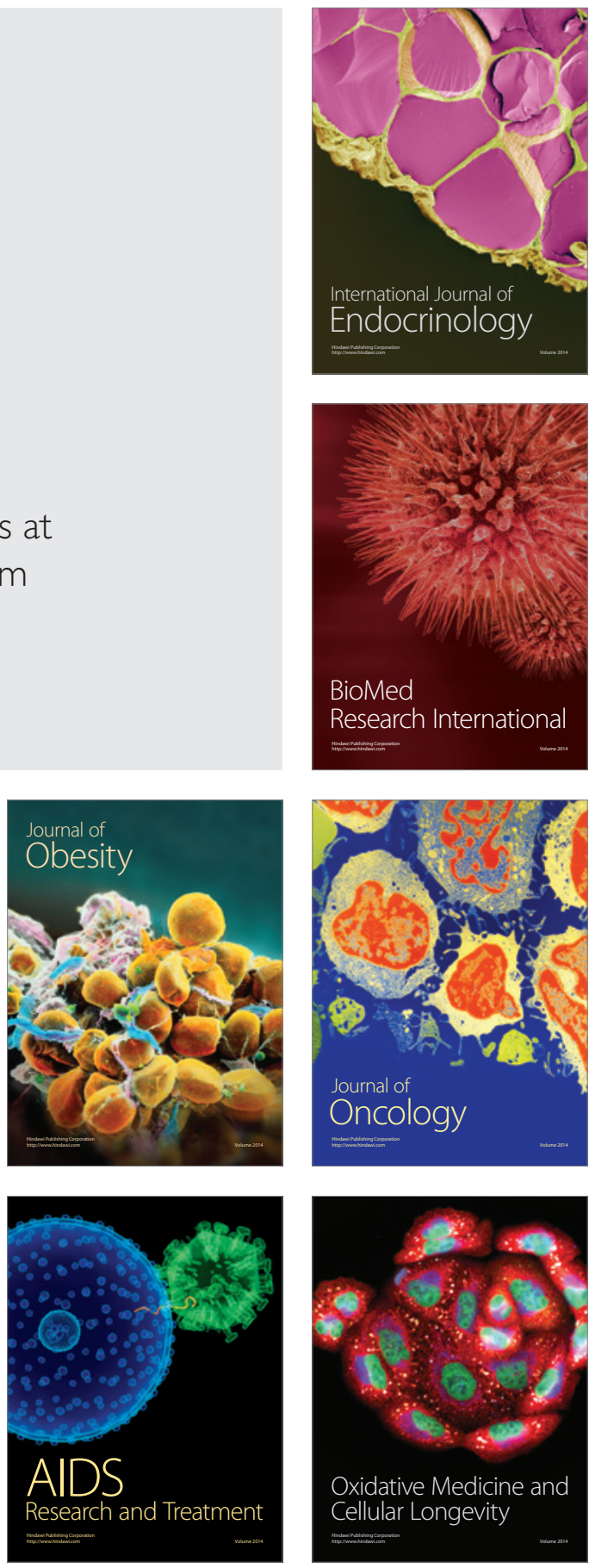